Swarthmore College

Works

\title{
Topic Maps Used To Present Interrelationships In Dynamic Linear Systems
}

\author{
Erik Allen Cheever , '82 \\ Swarthmore College, erik_cheever@swarthmore.edu
}

Ames Bielenberg , '13

Follow this and additional works at: https://works.swarthmore.edu/fac-engineering

Part of the Engineering Commons

Let us know how access to these works benefits you

\section{Recommended Citation}

Erik Allen Cheever , '82 and Ames Bielenberg, '13. (2011). "Topic Maps Used To Present Interrelationships In Dynamic Linear Systems". ASEE Annual Conference And Exposition. 22.1535.1-22.1535.10.

https://works.swarthmore.edu/fac-engineering/131

This work is brought to you for free by Swarthmore College Libraries' Works. It has been accepted for inclusion in Engineering Faculty Works by an authorized administrator of Works. For more information, please contact myworks@swarthmore.edu. 


\section{AC 2011-526: TOPIC MAPS USED TO PRESENT INTERRELATIONSHIPS}

IN DYNAMIC LINEAR SYSTEMS

Erik Cheever, Swarthmore College

Erik Cheever is a professor of Engineering at Swarthmore College. He teaches in the areas of Circuits, Electronics, Linear Systems, Control Theory and DSP.

Ames Bielenberg

Ames Bielenberg is an engineering student at Swarthmore College. 


\title{
Topic Maps Used to Present Interrelationships in Dynamic Linear Systems
}

\begin{abstract}
The study of linear physical systems is one of the topics in engineering with a rich interplay among the topics and no natural order in which to present the material. In this paper, we describe a system that uses topic maps to emphasize these interrelationships without imposing an arbitrary hierarchy on the material presented. For example, in terms of types of systems, mechanical systems can be taught first, or electrical. In terms of transforms, Laplace can be taught first, or Fourier, or even the Z-transform. There are also many relationships between topics. Examples include the transforms mentioned previously, the different types of physical system (electrical, mechanical, thermal...), and different solution methods such as state-space and transfer functions. By their very nature, textbooks must present and follow a linear path through the material, and any two textbooks are likely to follow very different paths. Web-based presentation of the material removes part of this problem because the material need not be presented linearly, but raises the prospect of a chaotic portrayal of the subjects because HTML links are merely from text to text and have no inherent meaning.
\end{abstract}

Another way to present such interrelated ideas is through the use of topic maps. Topic maps are used to represent these interrelationships using topics (concepts), associations (the relationships between topics) and occurrences (information, e.g., web pages, relating to the topics). This is distinct from concept maps and other commonly used visual methods for organizing information in that the ideas need not be hierarchical; for example Fourier Transforms are not a subset of Laplace transforms (or vice-versa) as is presented in most textbooks. In addition the topics have certain roles in the association that give the link meaning, a clear advantage over traditional HTML hyperlinks and other methods.

We have developed a system using HTML, PHP, JavaScript and XML that enables a user to author a topic map, as well as to present a graphical visualization of that map. When visualizing a map, a topic is selected (by clicking on it), and all related topics are displayed around it. Included in the display are the associations and roles played by each topic in that association. Topics can also have other information (occurrences in the form of HTML links, simulations...) that give explanatory detail about the topic, including descriptions of how it is related to other, associated, topics. While many textbooks (and even web-based presentations) have only short sections describing the interrelationships between topics, this system puts the relationship at the core of the presentation of the material to make it more clear why a particular topic is being learned and how it fits into the larger context of linear systems.

\section{Introduction}

At its lowest level the learning process, according to Bloom's taxonomy, involves moving a student from simply remembering information to understanding it $[1,2]$. The internet has greatly increased the amount and availability of information but has, in some ways, increased the difficulty of understanding that information. For example, using an internet search engine such as Google is a good way to get information, but it does not put the information in any kind of context for understanding. Semantic web technology has been developed to alleviate this 
problem by allowing computers to attach meaning to information. Tim Berners-Lee defines the semantic web as "a web of data that can be processed directly and indirectly by machines" [3]. It should be possible to use this machine-accessible "meaning" to develop computer-based systems that can help a student move from remembering to understanding.

Several technologies have been developed to exploit the semantic web; among them are OWL [4], RDF [5] and topic maps [6]. All have been used for educational purposes [7]. This paper focuses on the use of topic maps to encode and visualize the information that is typically contained in a Linear Systems course. Topic maps have been used previously for learning [8, 9 , $10,11,12]$, though not widely. There are several topic map editors and visualizers available, but these are generally for much larger systems and require Java to operate. The system described here has a much smaller footprint, runs quickly, and requires only JavaScript and PHP. The topic maps discussed in this paper are stored as XML files in the XTM format [13].

In addition to describing a system that allows the maps to be visualized (similar in some regards to existing systems $[8,14,15,16])$ it also shows how information about linear systems and other fields can be coded in topic maps. Visualization is especially important for understanding the material to students that might be classified as "visual" learners by Felder's index of learning styles [17].

\section{Background}

Topic maps are a way of representing a set of topics, the relationships between them, and information about them. The "topics" can be any idea or thing, the relationships are called "associations" and represent how the topics are related, and the extra information is referenced by "occurrences." In the associations, the topics have "roles." As an example, in a "family" association there could be one topic with "father" role, another as "mother," and one or more topics in "daughter" and "son" roles. Once the family association is made, information about the family can be determined about parents and children, siblings, and even such things as cousins in a larger map.

Topic mapping is similar to the much more widely known methodologies of concept mapping and mind mapping, but is relatively new and have not yet found wide use in educational settings. Topic maps differ from the others in several important aspects that should be useful for learning many engineering domains. Concept maps (and mind maps) are both mainly used to represent hierarchical information with the most important topic at the center (or top), and subtopics arranged in a tree. Topics become farther from the main topic as they become less important. Relationships between topics at different levels, or on different "branches" of the tree are hard to show. Topic maps, on the other hand, can naturally associate any two (or more) topics. In addition, while the links between topics in a concept map can be named, the topics themselves don't take on specific roles except insofar as the format of the concept map dictates. This is not a problem in a hierarchical map (i.e., "Mary" "is daughter of" "John") it becomes a problem in situations where the ideas form more of a web than a hierarchy. For example neither "state space" nor "transfer function" is below the other in a hierarchical sense, but they are both associated with each other. 
Another difference between concept maps and topic maps is that topics in topic maps by their very nature have additional information available in the form of occurrences. The occurrences can be brief (text or an image) or quite complex (a web page with examples and problems).

\section{A Simple Topic Map}

To demonstrate how these things work, consider the topic map depicted in figure 1 that shows a simple map for a building.

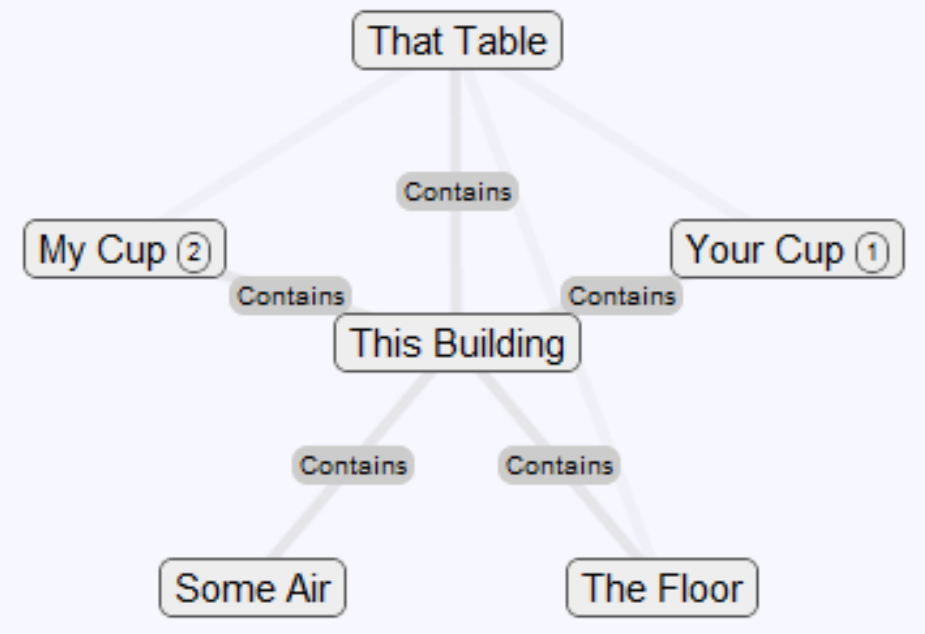

Figure 1: Topic map, with “This Building” at the center.

Note several things:

- "This Building" is the topic of interest. It is at the center of the map.

- Associations are shown radially, and are given from the perspective of the building (e.g., "This Building Contains That Table").

- Relationships at the next level are shown by a lighter line (e.g., the relationship between "My Cup" and "That Table."

- The number of occurrences associated with a topic are shown by a number in a circle ("My Cup" has 2 occurrences described below)

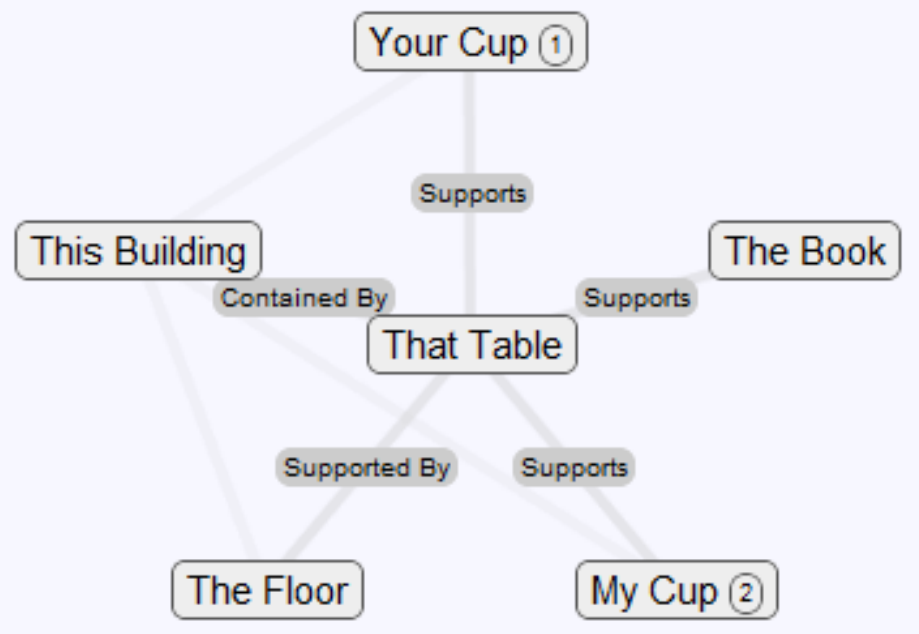

Figure 2: "That Table" is the topic of interest. 
Clicking on "That Table" makes it the topic of interest. See figure 2. Note that "That Table" is now at the center. Associations are shown from the perspective of the table. Note, in particular, that the associations between the table and the cups are now explicitly shown.

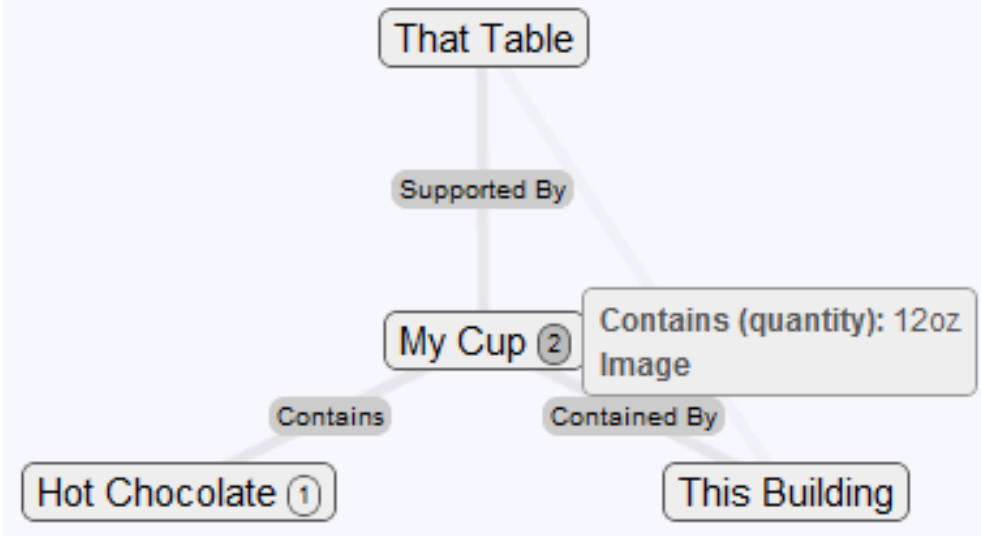

Figure 3: "My Cup” is topic of interest.

Figure 3 shows "My Cup" as the center topic. The circle has been clicked to show the occurrences associated with the topic of which there are two: its volume and a hyperlink ("Image" is a hyperlink to an picture of hot chocolate).

The current system displays only the topics that are directly associated with the topic of interest. Other systems exist that allow for the display of multiple levels [8, 14], but in learning systems it can be important to restrict the amount of information presented to the viewer so as not to overwhelm and, perhaps, confuse $[18,19]$ the reader.

The figures above show the visualization of the topic map. The diagram below shows the page used to edit the maps (the "My Cup" topic is being edited). 


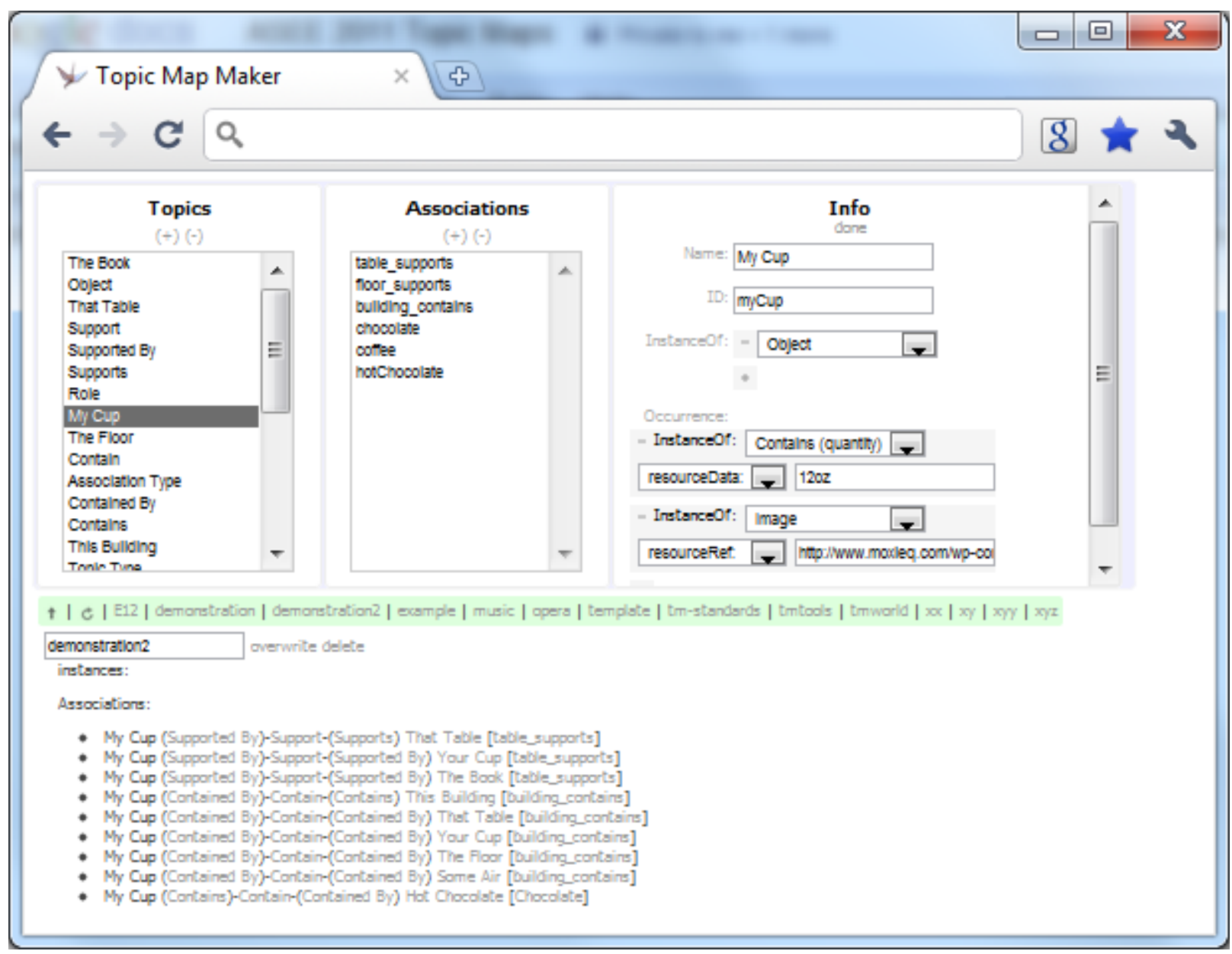

Figure 4: The topic map editor.

Obviously a topic map can be used to represent much more complex information than given by the previous example. For example, they have even been employed by the IRS to improve access to the tax code [20]. A good candidate for expression through topics maps is that of linear systems.

\section{A Topic Map for Linear Systems}

One field of engineering that is a good candidate for is that of linear systems because the information is highly interconnected, but not necessarily hierarchical. It is this rich context that makes it a good subject for adaptation to a semantic network. There are different ways of representing systems mathematically: differential equations, state space, transfer functions, polezero diagrams. There are different ways of solving the systems: Laplace transforms, the method of undetermined coefficients... There are different problem domains: electrical, mechanical, thermal, fluid... All of these can be taught alone, but all are interconnected and as students understand the connections they can move up Bloom's taxonomy to understanding and beyond.

That the information is not hierarchical is readily apparent upon examination of the table of contents of several linear systems textbooks. Table 1 shows the order of presentation of a subset of the topics as they are covered in several textbooks. 


\begin{tabular}{|l|l|l|l|}
\hline \multicolumn{1}{|c|}{ Karnopp [21] } & \multicolumn{1}{c|}{ Cochin [22] } & \multicolumn{1}{c|}{ Cha [23] } & \multicolumn{1}{c|}{ Ogata [24] } \\
\hline Electrical Systems & Laplace Transform & Mechanical Systems & Laplace Transform \\
Mechanical Systems & Transfer Functions & Electrical Systems & Mechanical Systems \\
State Space & Mechanical Systems & Transient Response & Transfer Function \\
Laplace Transform & Electrical Systems & Laplace Transform & State Space \\
Transient Response & Transient Response & Transfer Function & Electrical Systems \\
Transfer Functions & Frequency Response & Frequency Response & Transient Response \\
Frequency Response & State Space & State Space & Frequency Response \\
\hline
\end{tabular}

Table 1: Order of material in several textbooks.

Clearly the order of presentation of material is not critical, but is necessarily presented as such because a textbook is, by necessity, linear. Much of the challenge of learning the subject matter in a Linear Systems course is not only learning techniques and concepts, but also learning the interconnections. Topic maps may help in this regard, because the interconnection between topics is made obvious.

Consider the topic "Laplace Transform" shown in a topic map, below.

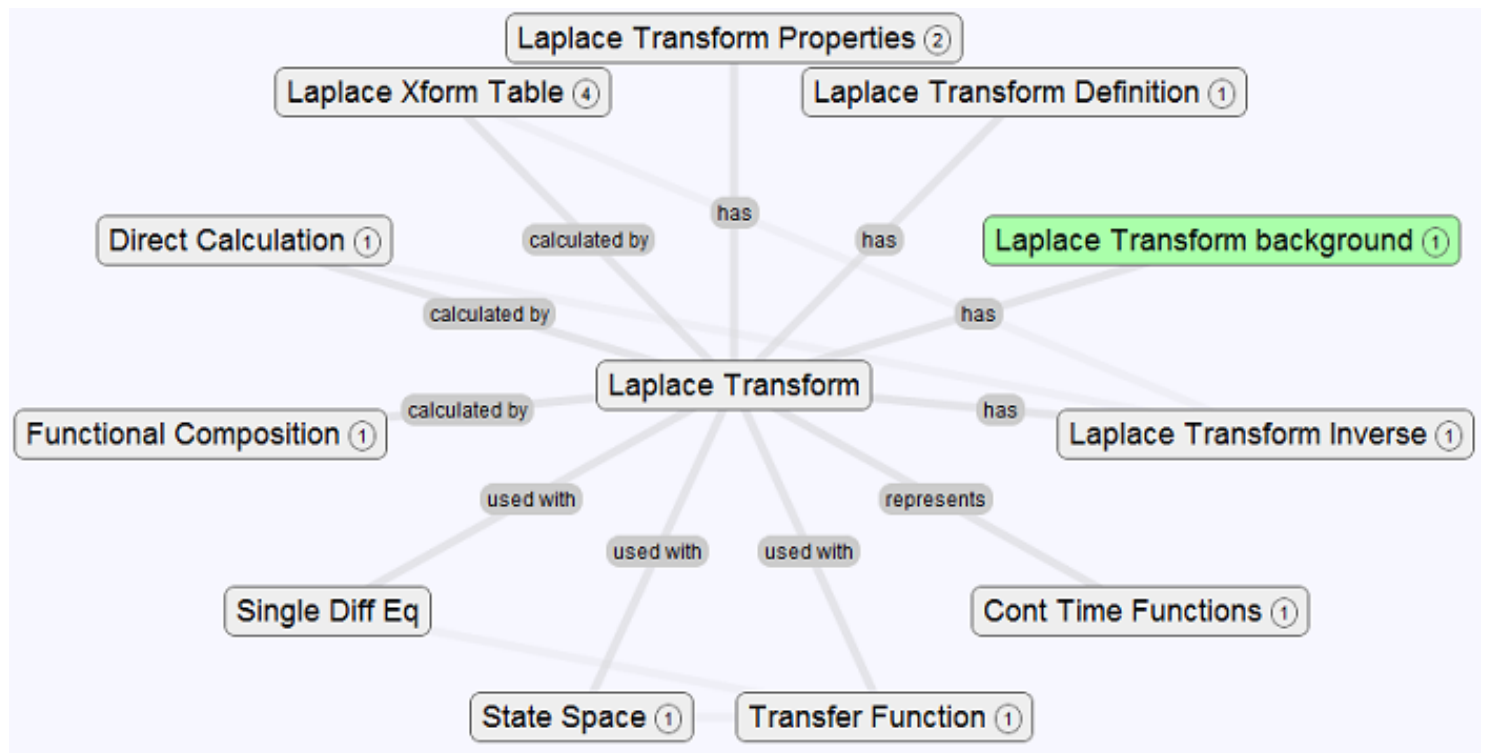

Figure 5: Topic map of "Laplace Transform"

The topic map immediately shows the context in which the Laplace transform is important. Some background information about the Laplace transform is highlighted (the student is likely to want to start here); the "Laplace Transform background" topic has an occurrence which is a web page describing background necessary to continue in the topic. Other topics are arranged around the main topic.

If, for example, the student wants to learn about the Laplace transform table s/he can select that topic and bring it to the center. Clicking on the encircled " 4 " reveals occurrences associated 
with the topic as shown in figure 6. In this case this is four links; one to a Laplace transform Table, another describing how the table is used, a third one with some examples, and a last one with problems to be completed.

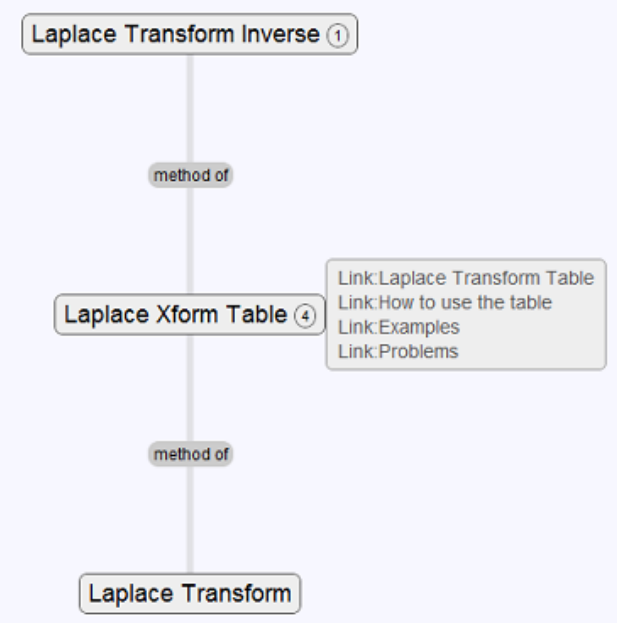

Figure 6: Topic of interest is “Laplace Xform Table,” and its occurrences are shown.

Likewise, starting from Figure 5 and selecting "Transfer Function" yields the map shown in figure 7 .

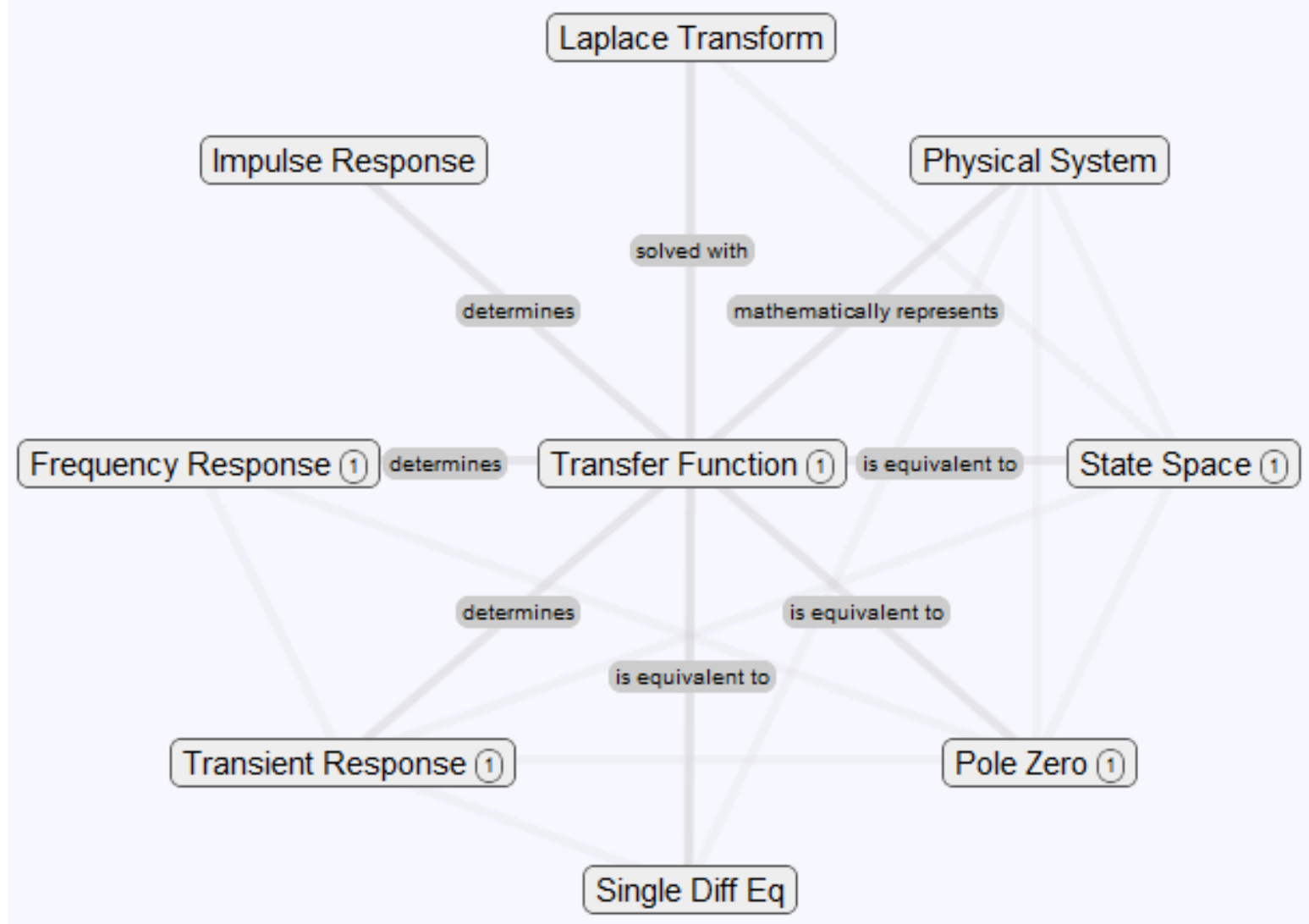

Figure 7: Topic map with “Transfer Function” at the center. 
This map clearly shows the relationship between the Transfer Function and other topics, but indicates that it is equivalent to the State Space representation.

\section{Conclusion}

A system has been developed to create and visualize topics maps. It can be deployed on any web server with PHP support; otherwise it requires only and JavaScript on the browser side. A topic map, along with supporting information in the form of web pages, was used represent the information in a typical Linear System Dynamics course. Instead of presenting the information to the student in a linear manner, the topic map puts the information immediately in context in an explicit a visual way. Learning must still occur one topic at a time, but the order of topics is not preordained, and the student is constantly being presented the "big picture" as s/he navigates through the topic map.

Linear systems are not the only field that might benefit from description as a topic map. As noted, its use in educational contexts $[8,19]$ is not novel. It has been used for highly interconnected topics as disparate as Chinese herbs [11], Italian Opera [25], and the US tax code [20]. Many other fields within engineering also have concepts that are highly interconnected. For example control theory has several types of controllers (PI, lead...), domains (continuous time, discrete time, Laplace, Z...), techniques (root locus, Nyquist, Bode), that could be represented, along with their associations, by a topic map.

As the map is deployed in a Linear Systems course, students learning will be assessed as to whether the topic map helped in learning the material, and whether they have a better grasp of the "big picture."

The map discussed in this paper is available at http://www.swarthmore.edu/NatSci/echeeve1/Ref/LPSA/TM/tmExplore/index.html?LPSA\#t_lpsahome.

\section{References}

[1] Taxonomy of Educational Objectives, Handbook I: The Cognitive Domain, Bloom, David McKay Co Inc., 1956

[2] A taxonomy for learning, teaching and assessing: A revision of Bloom's Taxonomy of educational outcomes, Anderson \& Krathwohl, Longman, 2001

[3] “P2P Foundation (en)," http://p2pfoundation.net/Semantic_Web, (accessed January 6, 2011)

[4] “ OWL 2 Web Ontology Language Document Overview," http://www.w3.org/TR/owl2-overview/, (accessed January 7, 2011)

[5] "Semantic Web Standards (en)," http://www.w3.org/RDF/, (accessed January 7, 2011)

[6] "TopicMaps.org - Topic Maps," http://www.topicmaps.org/, (accessed January 7, 2011)

[7] "Use of the semantic web to solve some basic problems in education," Koper, Journal of Interactive Media in Education, 6, 2004.

[8] "TM4L: Creating and browsing educational topic maps," Dicheva \& Dichev, British Journal of Educational Technology, 37:3, 2006, pp 391-404.

[9] “Using Topic Maps for E-Learning," Dichev, Dicheva \& Aroyo, Proceedings of the IASTED International Conference on Computers and Advanced Technology in Education, 2003.

[10] "Designing interactive learning environments: an approach from first principles," Scott \& Cong, Campus Wide Information Systems, 24:3, 2007, pp 174-286. 
[11] "Organizing learning materials through hierarchical topic maps: an illustration through Chinese herb medication," Shih, Shih \& Chen, Journal of Computer Assisted Learning, 23, 2007, pp 477-490.

[12] “A Topic Map based Adaptive Tutoring System," Manitsaris \& Hatzigaidas, The 5th Int.Conf on Applied Informationc and Communications, 2005, pp 264-269.

[13] XML Topic Maps: Creating and Using Topic Maps for the Web, Park \& Hunting, Addison-Wesley Professional, 2002

[14] “[Omnigator] Welcome Page,” http://www.ontopia.net/omnigator/models/index.jsp, (accessed January 7 , 2011).

[15] "Information Management - Topic Maps Visualization," Le Grand \& Soto, XML Europe 2000, http://www.gca.org/papers/xmleurope2000/papers/s29-03.html

[16] "Visualisation of the Semantic Web: Topic Maps Visualisation," Sixth International Conference on Information Visualisation (IV'02), 2002, pp.344-349.

[17] "Learning Styles and Teaching Styles in Engineering Education," Felder \& Silverman. Engineering Education, 78:7, 1988, 674-681.

[18] Debunking the Buzz Words, Or Can Hermeneutic Analysis Be Used To Evaluate Pedagogically Based Learning Objects Designed from Constructivist Epistemological Ontologies Defined in XML Metadata?, Stuckey, Bronwyn; Hensman, Jim; Hofmann, Tobias; Dewey, Barbara; Brown, Helen; Cameron, Sonja, ED-MEDIA 2002

World Conference on Educational Multimedia, Hypermedia \& Telecommunications, 2002

[19] Dicheva, D. "TM4L: Creating and browsing educational topic maps." British journal of educational technology 37.3 (2006): 391-404.

[20] "IRS Tax Map," http://taxmap.ntis.gov/taxmap/, (accessed January 6, 2011)

[21] System Dynamics, Karnop, Margolis \& Rosenberg, John Wiley and Sons, 2006.

[22 Analysis and Design of Dynamic Systems, Cochin \& Cadwallender, Addison Wesley, 1997.

[23] Fundamentals of Modeling and Analyzing Engineering Systems, Cha, Rosenberg \& Dym, Cambridge University Press, 2000.

[24] System Dynamics, Ogata, Prentice Hall, 2004.

[25] "OperaMap: The Italian Opera Topic Map," http://www.ontopia.net/operamap/index.jsp, (accessed January 11, 2011). 